\title{
Adherence to metformin is reduced during school holidays and weekends in children with type 1 diabetes participating in a randomised controlled trial
}

\author{
Catherine Leggett, ${ }^{1}$ Lynne Giles, ${ }^{2}$ Jemma Jay Angela Anderson, ${ }^{3,4}$ Matthew Doogue, ${ }^{5}$ \\ Jennifer Couper, ${ }^{3,4}$ Alexia Sophie Pena ${ }^{\circledR 3,4}$
}

'SA Pharmacy, Women's and Children's Hospital, North Adelaide, South Australia, Australia

${ }^{2}$ School of Public Health, University of Adelaide, Robinson Institute, Adelaide, South Australia, Australia

${ }^{3}$ Endocrinology and Diabetes Department, Women's and Children's Hospital, North Adelaide, South Australia, Australia

${ }^{4}$ Discipline of Paediatrics, University of Adelaide, Robinson Research Institute, Adelaide, South Australia, Australia ${ }^{5}$ Department of Medicine, University of Otago, Christchurch, New Zealand

\section{Correspondence to} Catherine Leggett, SA Pharmacy, Women's and Children's Hospital, North Adelaide, South Australia 5006, Australia;

catherine.leggett@sa.gov.au

Received 27 September 2018 Revised 16 March 2019

Accepted 24 April 2019 Published Online First 11 May 2019

\section{ABSTRACT}

Background Non-adherence to treatment in childhood chronic illness has serious consequences for health and healthcare costs. Accurate detailed objective data on adherence are minimal in this age group.

Objective To evaluate medication adherence using electronic monitoring systems in children with type 1 diabetes (T1D).

Design A cohort study of 90 T1D children (aged 13.6 2.5 years, 41 males) from two paediatric diabetes clinics, participated in a 12-month double-blind, randomised, placebo-controlled trial (1:1 allocation). This cohort provided 28336 days of study observations; 7138 school holiday and 8875 weekend/public holiday days. Method Adherence to intervention (metformin $(n=45)$ or placebo $(n=45))$ was measured objectively by Medication Event Monitoring Systems (MEMS) including proportion of medication doses taken and daily adherence patterns and by tablet count at 3, 6 and 12 months. The trial was completed in June 2015.

Results There was an average (SD) of 363.3 (42) days of MEMS observations available for each study participant (94.1 (12.6) school holiday days and 117.1 (13.4) weekend/public holiday days). Adherence reduced during school holidays (adjusted OR (aOR) $0.81 ; 95 \% \mathrm{Cl}$ 0.72 to $0.91 ; p<0.001)$ and during weekends/public holidays (aOR 0.74; 95\% Cl 0.69 to $0.80 ; p<0.001$ ). Adverse effects to the intervention did not affect overall adherence (aOR 0.77; 95\% Cl 0.3 to $2.01 ; p=0.6$ ). Age, gender, body mass index, diabetes duration, insulin dose, HbA1c (Haemoglobin A1c) or socioeconomic status did not predict adherence.

Conclusion Medication adherence was reduced during school holidays and on weekends in children with T1D. Clinical characteristics including socioeconomic status and the presence of adverse effects did not predict adherence.

Trial registration number ACTRN12611000148976.

\section{INTRODUCTION}

Chronic non-communicable diseases, including type 1 diabetes (T1D), are increasingly prevalent in childhood, ${ }^{12}$ and treatment is becoming more complex with combination therapies being common. Non-adherence to medical treatment in childhood has serious consequences for an individual's health and substantially increases demand and expenditure on health systems. ${ }^{3}$ Limited available data suggest poor adherence is common in children with chronic conditions and during adolescence. ${ }^{4-6}$
What is already known on this topic?

- Non-adherence to medical treatment in children with chronic disorders has serious consequences for health and healthcare costs.

- Adherence as measured by prescription refill data is suboptimal in children and adolescents with chronic disorders.

\section{What this study adds?}

Adherence to a long-term intervention, measured by an electronic system, is reduced during holidays and weekends in children with type 1 diabetes.

- The presence of adverse effects to intervention (metformin) did not predict adherence.

However, most studies use pharmacy prescription refill data or tablet count as adherence measurements.

Tablet count has been commonly used as a measure of adherence in children with T1D. ${ }^{7-9}$ This method provides a single average measure of adherence and does not account for specific tablet-taking behaviours. ${ }^{1011}$ Tablet count overestimates adherence as data are easily altered by discarding tablets. Electronic dose monitoring devices such as Medication Event Monitoring Systems (MEMS) passively and prospectively record the date and time an individual accesses their medications, providing a more reliable objective and validated continuous dosing history. ${ }^{11} 12$ The Adelaide Metformin Trial provided a unique opportunity to evaluate adherence patterns using MEMS in children with T1D over an extended period compared with previous studies. ${ }^{7-9}$ This randomised controlled trial (RCT) evaluated the effect of metformin in addition to insulin therapy on vascular health over a 12-month period. Our hypotheses were that: (1) children with T1D are less adherent during school holidays and (2) those with better baseline glycaemic control have higher adherence. We aimed to evaluate adherence patterns to the intervention using objective measurements in children enrolled in the RCT over 12 months. 


\section{METHODS}

\section{Study design and participants}

This study prospectively evaluated adherence patterns in 90 children (aged 8-18 years) with T1D participating in a double-blind, randomised, placebo-controlled trial investigating the effects of metformin on vascular health. Participants were randomised to receive oral metformin or placebo (1:1 allocation) for 12 months with no systematic change in insulin treatment. ${ }^{13} 14$ Participants were recruited consecutively by investigator JJAA from two paediatric diabetes centres in Adelaide, South Australia. Pharmacy subsequently assigned participants to intervention or placebo using a generated list. Participants were eligible if they were diagnosed with T1D at least 6 months prior, had an insulin requirement $>0.5 \mathrm{U}$ insulin $/ \mathrm{kg} /$ day, aged $8-18$ years and a body mass index $(\mathrm{BMI})>50$ th percentile for age and sex. ${ }^{15}$ They were excluded if they had a severe hypoglycaemic episode within 6 months prior to recruitment, more than two episodes of diabetic ketoacidosis in the previous 12 months, serious comorbidities, contraindication to metformin therapy or were already on metformin, statins, multivitamins or antihypertensives. ${ }^{14}$ The trial was prospectively registered with the Australia and New Zealand Clinical Trials Registry. Participants were recruited from August 2011, and the trial was completed once the sample size was reached (June 2015). Written informed consent was obtained from all parents, and written assent was obtained from all participants.

\section{Assessments}

Adherence

Adherence to the medication was evaluated prospectively over 12 months by two methods: (1) electronic dose monitoring with MEMS caps (AARDEX group, LTD Sion, Switzerland) and (2) by tablet count at each assessment time point after baseline ( 3 months, 6 months and 12 months); unused medication was counted and compared with the number of prescribed doses during each period. Strategies to improve adherence at each visit were individualised for each participant specific to their needs and visits occurred mainly during the week. The MEMS data since last visit were reviewed with each participant in conjunction with their tablet count. Overall adherence was discussed as well as specific times when tablets were not taken. This approach was not different in school holidays versus school term.

Adherence was assessed as short term (0-3 months), medium term (3-6 months) and long term (6-12 months). Average adherence was calculated across the three assessment periods, and each participant was then classified as optimally adherent or not on the basis of average adherence at each time period and overall. Optimal adherence was defined as $\geq 80 \%$ of prescribed doses taken over a defined period. ${ }^{16} 17$

\section{Socioeconomic status (SES)}

SES was defined on the basis of state-based deciles in the Socioeconomic Index for Areas (SEIFA) Index of Relative Social Disadvantage according to participant's postcode at study baseline. ${ }^{18}$ The 2011 SEIFA index was used as this was nearest to the time of first participant recruitment. For the present study, SES was defined as low (state decile 1-3), medium (state decile 4-7) or high (state decile 8-10).

\section{Adverse effects}

Adverse effects were obtained through completion of a telephone questionnaire conducted via fortnightly phone calls (JJAA) for the first 3 months and monthly phone calls thereafter as well as at assessments at 3, 6 and 12 months. Adverse effects in the questionnaire included rash, hypoglycaemia (moderate or severe), diabetic ketoacidosis, gastrointestinal symptoms (specifically nausea, vomiting, diarrhoea and anorexia) and any other adverse effects reported. ${ }^{13}$

\section{Statistical analyses}

MEMS caps recorded the number of episodes of bottle opening for each participant, and the data were downloaded for analysis. MEMS adherence was defined as the number of bottle openings in a 24-hour period divided by the number of prescribed doses. Calculations for adherence were made for every calendar day with a 03:00 cut-off. For a given participant, an absence of bottle openings on any day (ie, 'missing' MEMS episodes) was classified as non-adherent for that particular day. MEMS adherence was evaluated daily, aggregated across each assessment period, and overall across the entire 12 months.

The difference in adherence between three successive assessment periods (ie, $0-3,3-6$ and 6-12 months) was also calculated for each participant, and the median decrease across the entire follow-up period was then derived from these differences. Mixed effects linear regression models using all available data were fit to investigate the effects of treatment group and assessment period (ie, 0-3, 3-6 and 6-12 months) on adherence, with separate models for MEMS and tablet count. Likelihood ratio tests were used to ascertain the statistical significance of treatment and assessment period.

MEMS adherence patterns were calculated for each participant from baseline to the final follow-up visit. Each day in the study period was classified as school term (including weekends) or school holidays, according to the South Australian Department of Education and Child Development published state school term dates across 2011 through 2015. ${ }^{19}$ Separate to this, each day of the week was classified as a weekday (Monday through Friday) or a weekend (Saturday or Sunday) or public holiday. Public holidays were extracted from the South Australian Government's listing of official public holidays. ${ }^{20}$

The effect of school holidays on daily adherence patterns was evaluated using mixed effects logistic regression models, adjusted for age, gender and intervention and with a random effect for participant and robust variance estimator. For each participant, all available data were used in the analysis of adherence patterns so as to account for time in study. ORs and 95\% CIs were used to summarise results. Sensitivity analyses that included indicator variables for weekends or public holidays were also conducted, so as to assess whether public holidays or weekends changed any observed effect of school holidays on adherence. Analyses were also adjusted for baseline HbA1c and SES.

A logistic regression model was used to investigate the association between overall adherence and adverse effects over the 12 -month study period. Mean adherence was dichotomised based on an $80 \%$ cut point and adverse effects classified as present or absent. The model was adjusted for age, gender, intervention and $\mathrm{HbA} 1 \mathrm{c}$. Other potential predictors of overall adherence considered were BMI, diabetes duration, insulin dose or SES. Gender, intervention and SES were treated as categorical variables in the statistical models and the remaining predictors were included as continuous variables.

Pearson's correlation coefficients were calculated to assess the relationship between MEMS and tablet count in each assessment period and over 12 months. Correlations were calculated for all available data in each assessment period and then separately for the metformin and placebo groups. Bland-Altman limits of 


\begin{tabular}{llllll}
\hline $\begin{array}{l}\text { Table } 1 \\
\text { study }\end{array}$ & Adherence data using MEMS and tablet count over the \\
\cline { 1 - 2 } $\begin{array}{l}\text { Assessment } \\
\text { period } \\
\text { (months) }\end{array}$ & Median & $95 \% \mathrm{Cl}$ & & Median & $95 \% \mathrm{Cl}$ \\
\cline { 2 - 3 } \cline { 5 - 6 } $0-3$ & 88.9 & 77.0 to 91.6 & & 87.1 & 78.2 to 90.5 \\
\hline $3-6$ & 78.8 & 69.9 to 83.2 & & 78.6 & 70.4 to 84.2 \\
\hline $6-12$ & 67.5 & 60.0 to 74.8 & & 72.4 & 66.4 to 79.2 \\
\hline $12^{*}$ & 75.5 & 65.7 to 81.5 & 76.6 & 69.7 to 80.5 \\
\hline
\end{tabular}

*Average MEMS or tablet count values across 12 months calculated using all available data.

MEMS, Medication Event Monitoring Systems.

agreement (LoAs) for MEMS and tablet count over 12 months were also derived overall and separately for the metformin and placebo groups.

Statistical significance was set at $\mathrm{p}<0.05$ (two sided). All analyses were conducted according to a prespecified analysis plan and used Stata V.14.1.

\section{RESULTS}

Ninety children (age 13.6 \pm 2.5 years, 88 Caucasians, 41 male, 45 per intervention group) with T1D (median duration 4.4 years, IQR 2.5-8.1 years, range $0.6-16.2$ years, 45 on continuous subcutaneous insulin infusion and 45 on multiple daily insulin injections) participated in the RCT. At baseline, the median (IQR) HbA1c was $8.7 \%(8.1-9.9) / 72 \mathrm{mmol} / \mathrm{mol}(65-85)$ and mean (SD) BMI z-score was 0.89 (0.57). ${ }^{14}$ Baseline demographics and clinical characteristics of each group have been previously reported. ${ }^{14}$ The distribution of SES was low (26), mid (22) and high (42). Tablet adherence data were available for 81 children (eight withdrew, one did not return tablets for counting). MEMS adherence data were available for 78 children (eight withdrew, four did not use MEMS).

Adherence reduced over the three assessment periods according to MEMS and tablet count $(\mathrm{p}<0.001$ and $\mathrm{p}<0.001$, table 1$)$ with no difference between metformin and placebo groups in the decrease in adherence ( $p>0.1$ for MEMS and tablet count). Adherence by MEMS decreased by a median of 5.5\% (95\% CI $2.7 \%$ to $9.1 \%$ ) between 3 and 6 months, 5.5\% (95\% CI 3.6\% to $9.0 \%$ ) between 6 and 12 months and 9.6\% (95\% CI 4.4\% to $18.2 \%)$ across the entire follow-up period, with similar decreases observed for tablet count (median decrease: 3-6 months: 4.8\%, $95 \%$ CI $0.8 \%$ to $10.0 \%$; $6-12$ months: $4.6 \%, 95 \%$ CI $2.3 \%$ to $7.8 \%$; across the entire follow-up period: $12.1 \%, 95 \%$ CI $5.4 \%$ to $17.4 \%)$.

MEMS and tablet count adherence measures were highly correlated at each assessment period (0-3 months: $r=0.96$, 3-6 months: $\mathrm{r}=0.89$ and $6-12$ months: $\mathrm{r}=0.91$, all $\mathrm{p}<0.0001$ ) and over 12 months $(\mathrm{r}=0.95, \mathrm{p}<0.0001$, figure $1 \mathrm{~A})$ with no significant difference between intervention groups observed (all $\mathrm{p}>0.05$ ). The mean difference between MEMS and tablet count within individuals was 1.4 (95\% CI -0.3 to 3.0$)$, and this was similar when separately calculated for placebo $(1.1 ; 95 \% \mathrm{CI}$ -1.5 to 3.8$)$ and metformin groups $(1.7 ; 95 \% \mathrm{CI}-0.3$ to 3.6$)$. The associated LoAs were -13.5 (95\% CI -16.0 to -11.2 ) to 16.2 (95\% CI 14.0 to 18.8 ) (figure $1 \mathrm{~B}$ ), again similar when calculated separately for placebo (mean difference 1.1; LoA -16.1 (95\% CI -20.7 to -12.9 ) to 18.4 (95\% CI 15.2 to 23.0$)$ ) and metformin groups (mean difference 1.7; LoA - 9.9 (95\% CI -13.4 to -7.7$)$ to $13.2(95 \%$ CI 11.0 to 16.7$)$ ). Median adherence by MEMS over 12 months was $75.5 \%$ (95\% CI 65.7 to
A

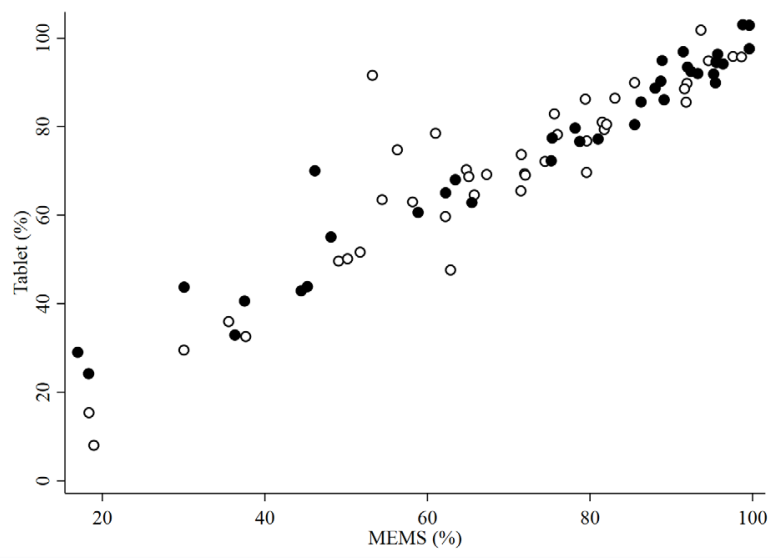

B

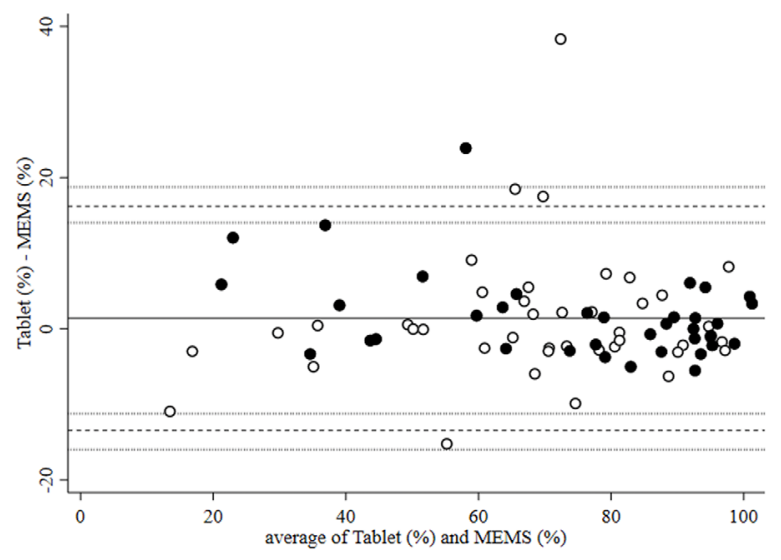

Figure 1 Association between adherence methods over 12 months. (A) Scatter plot of tablet versus MEMS (\%), r=0.95, $p \leq 0.0001$. (B) Bland-Altman plot of adherence measurements (tablet and MEMS (\%)) over 12 months. Solid line indicates mean difference, dashed lines indicate limits of agreement $(-)$ and dotted lines indicate $95 \% \mathrm{Cl}$ for limits of agreement (...) between the two methods of measurement. Open circles indicate placebo and closed circles metformin. MEMS, Medication Event Monitoring Systems.

81.5). Figure 2 presents examples of MEMs adherence patterns during the study (figure part 2A: optimal adherence over first 3 months of the study, figure part 2B: less than optimal adherence from 3 to 6 months and figure part 2C less than optimal adherence 6-12 months).

A total of 28336 days of observation were included in the adherence pattern analyses, and an average of 363.3 days (SD 41.7) per participant contributed to the analyses. There were 7138 school holiday days (25\%) in the observation period, with an average of 94.1 days (SD 12.6) per child and 8875 weekend/ public holiday days (31.3\%), with an average of 117.1 days (SD 13.4) per child. The odds of adherence were reduced by $19 \%$ during school holidays (OR $0.81 ; 95 \%$ CI 0.72 to 0.91 ; $\mathrm{p}<0.001)$ compared with school term time. The effect of school holidays remained when adjusted for age, gender, SES, HbA1c and intervention group (adjusted OR (aOR) 0.81; 95\% CI 0.72 to $0.91 ; \mathrm{p}<0.001)$. Sensitivity analyses showed that this effect was unchanged when weekends (aOR 0.83 ; 95\% CI 0.74 to $0.93 ; \mathrm{p}<0.001$ ) or public holidays (aOR $0.83 ; 95 \%$ CI 0.74 to $0.93 ; \mathrm{p}=0.001)$ were included in the statistical models. Weekends and public holidays were independently associated with a $26 \%$ reduction in odds of adherence (aOR $0.74 ; 95 \%$ CI 0.69 to $0.80 ; \mathrm{p}<0.001)$. 
a) 0 - 3 months ( $89 \%$ adherence)

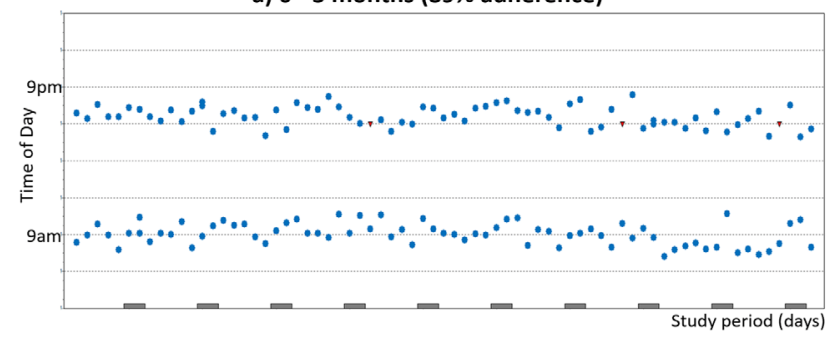

b) 3 - 6 months (79\% adherence)

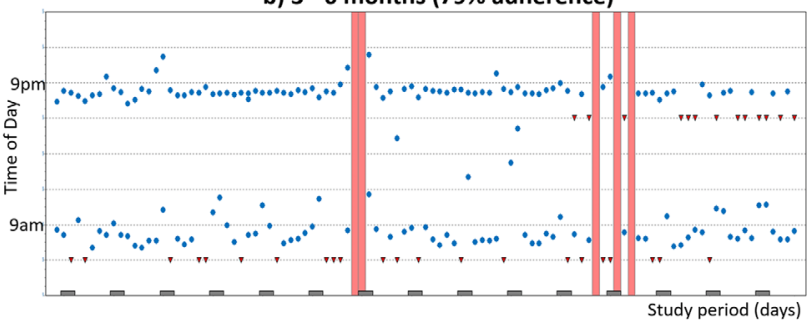

c) 6 - 12 months (65\% adherence)

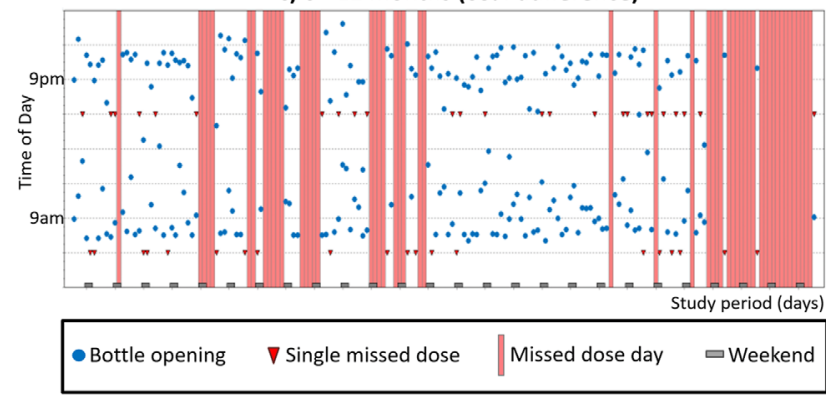

Figure 2 Illustrations of individual MEMS adherence patterns for three study periods. MEMS, Medication Event Monitoring Systems.

Baseline age, gender, BMI z-score, diabetes duration, insulin dose, HbA1c or SES did not predict adherence over 12 months (all p values $>0.05$ ). Adverse effects were reported by $45 / 90$ children (24 metformin and 21 placebo). There was no difference in the number of reported side effects between groups (median adverse effects per participant in the metformin group was 1 (95\% CI 0 to 2$)$ and in the placebo group was 0 (95\% CI 0 to 1$)(p=0.5)$. The presence of any adverse side effects over 12 months did not affect overall adherence (aOR 0.77 95\% CI 0.3 to $2.01, \mathrm{p}=0.6$ ).

\section{DISCUSSION}

In this 12-month study, children with T1D had reduced adherence to an additional oral intervention during school holidays and weekends/public holidays compared with school terms and week days. Age, gender, BMI, glycaemic control, SES and/or the presence of adverse effects did not predict adherence. To our knowledge, this is the first study reporting decreased adherence to treatment during less structured periods such as school holidays in children with T1D. A similar pattern was found in a study of adherence to nebulised interventions in children with cystic fibrosis, ${ }^{21}$ which suggests this pattern of adherence may extend to other chronic diseases.

As school holidays and weekends account for 25\% and 30\%, respectively, of a calendar year in Australia, the impact of our findings are considerable. Furthermore, a 25\% reduction in adherence has been associated with significant increases in hospitalisation and mortality in adults with T1D. ${ }^{22}$ Targeted reminders and additional strategies to improve adherence during these less structured periods for school children are necessary to optimise benefit from their treatment.

Our finding that adherence was not predicted by demographic or clinical characteristics at baseline is consistent with a meta-analysis of adherence in large cohort of children and adolescents with T1D. ${ }^{23}$ This supports the concept of adherence being multifactorial and shows that assumptions cannot be made about patients' adherence. Other factors that were not evaluated in the meta-analysis or in our study such as self-care, disturbed eating behaviour, depression, number of blood glucose levels taken and parental involvement may also impact on adherence. ${ }^{24}{ }^{25}$ We did not find a relationship between baseline HbA1c and adherence as previously described in a meta-analysis with a large sample size. ${ }^{23}$ Additionally our study evaluated adherence to metformin or placebo not insulin therapy.

While MEMS offers the advantage of obtaining specific patterns related to dose-taking behaviours, our study also found MEMS and tablet count were highly correlated at each visit and over 12 months. Although LoAs were wide, the differences between MEMS and tablet count within individuals were $1.4 \%$ on average and less than $10 \%$ in $90 \%$ of participants. This suggests that tablet count can provide a reasonable and less expensive measurement of adherence in children and adolescents with T1D and may be sufficient for measuring overall adherence. The overall level of adherence of $75.5 \%$ in our study over 12 months was comparable with a large multicentre trial of statins and ACE inhibitors in adolescents with $\mathrm{T}^{2} \mathrm{D}^{26}$ and to other studies of shorter duration using metformin in children with T1D. ${ }^{7-9}$ Previous metformin studies have used tablet count as an adherence measurement and only reported an average adherence measurement over the entire study period. We detected a decline in adherence over the 12 months with optimal adherence $(>80 \%)$ over the first 3 months of intervention. Decline in adherence was also reported in a trial of longer duration in adolescents T1D but using more additional oral interventions. ${ }^{26}$

There are a number of strengths in our study. First, the use of MEMS that records dosing dates and times allowing evaluation and further understanding of tablet taking behaviour. This detailed information from MEMS identified patterns of adherence that will assist in the development of strategies to enhance adherence in children using long-term medications. ${ }^{27}$ Second, the study had a longer duration than other trials in which adherence to metformin in children with T1D has been assessed. ${ }^{7-9}$ Third, the studied population included children with different levels of diabetes control, different insulin delivery methods and from across the socioeconomic spectrum. Limitations of the study include that the intervention evaluated was in addition to the daily routine of insulin treatment, which may affect individuals' adherence to other treatments. Furthermore, our sample size of 90 was relatively small but offset by the detailed electronic evaluation of adherence over the 12 months including more than 28000 observations. A final limitation is that the participants were predominantly Caucasian, reflecting the composition of our South Australian T1D clinic population, and our results may not be generalisable to other ethnic groups.

\section{CONCLUSION (IMPLICATIONS)}

In summary, we demonstrated an approximate 20\% reduction in treatment adherence during school holidays and weekends/ public holidays in children with T1D. Clinicians should be alert to this temporal trend when managing children with chronic conditions and develop targeted strategies to enhance adherence 
during vulnerable periods in these children, improving overall quality of healthcare.

Acknowledgements The authors would like to thank Dr Veronica Corotto for her assistance with organising data and all participants in the study for their involvement.

Contributors $\mathrm{CL}$ drafted the manuscript and had full access to the study data and acquired, analysed and interpreted the data and takes responsibility for the integrity of the contents of the article, the data and the accuracy of the data analysis. LG drafted the manuscript, provided statistical analysis and had full access to all of the data in the study and take responsibility for the integrity of the contents of the article, the data and the accuracy of the data analysis. JJAA obtained funding for this study and had full access to the study data and acquired, analysed and interpreted the data and takes responsibility for the integrity of the contents of the article, the data and the accuracy of the data analysis. MD drafted the manuscript and acquired, analysed and interpreted the data. ASP and JC obtained funding for this study, drafted the manuscript and had full access to the study data and acquired, analysed and interpreted the data and take responsibility for the integrity of the contents of the article, the data and the accuracy of the data analysis. All authors critically reviewed the manuscript for important intellectual content and approved the final version for publication.

Funding This study was funded by Diabetes Australia Research Trust, Women's and Children's Foundation research project grants and Australasian Paediatric Endocrine Care grants. JJAA held an MS McLeod Research Foundation fellowship.

Disclaimer The funders had no role in the design and conduct of the study; collection, management, analysis and interpretation of the data; preparation, review or approval of the manuscript; and decision to submit the manuscript for publication.

Competing interests None declared.

Patient consent for publication Not required.

Ethics approval The trial was approved by the Women's and Children's Hospital Research Ethics Committee (HREC 2327/12/13) and Flinders Medical Centre Research Ethics Committee (HREC 443.12)

Provenance and peer review Not commissioned; externally peer reviewed.

\section{REFERENCES}

1 Van Cleave J, Gortmaker SL, Perrin JM. Dynamics of obesity and chronic health conditions among children and youth. JAMA 2010;303:623-30.

2 Berhan Y, Waernbaum I, Lind T, et al. Thirty years of prospective nationwide incidence of childhood type 1 diabetes: the accelerating increase by time tends to level off in Sweden. Diabetes 2011;60:577-81.

3 McGrady ME, Hommel KA. Medication adherence and health care utilization in pediatric chronic illness: a systematic review. Pediatrics 2013;132:730-40.

4 DiMatteo MR. Variations in patients' adherence to medical recommendations: a quantitative review of 50 years of research. Med Care 2004;42:200-9.

5 Morris AD, Boyle DIR, McMahon AD, et al. Adherence to insulin treatment, glycaemic control, and ketoacidosis in insulin-dependent diabetes mellitus. Lancet 1997:350:1505-10.

6 Gandhi K, Vu BK, Eshtehardi SS, et al. Adherence in adolescents with Type 1 diabetes: strategies and considerations for assessment in research and practice. Diabetes Manag 2015;5:485-98.
7 Libman IM, Miller KM, DiMeglio LA, et al. Effect of metformin added to insulin on glycemic control among overweight/obese adolescents with type 1 diabetes: a randomized clinical trial. JAMA 2015;314:2241-50.

8 Nadeau KJ, Chow K, Alam S, et al. Effects of low dose metformin in adolescents with type I diabetes mellitus: a randomized, double-blinded placebo-controlled study. Pediatr Diabetes 2015:16:196-203.

9 Hamilton J, Cummings E, Zdravkovic V, et al. Metformin as an adjunct therapy in adolescents with type 1 diabetes and insulin resistance: a randomized controlled trial. Diabetes Care 2003;26:138-43.

10 Osterberg L, Blaschke T. Adherence to medication. N Eng/ J Med 2005:353:487-97.

11 El Alili M, Vrijens B, Demonceau J, et al. A scoping review of studies comparing the medication event monitoring system (MEMS) with alternative methods for measuring medication adherence. Br $J$ Clin Pharmacol 2016;82:268-79.

12 Vrijens B, Urquhart J. Methods for measuring, enhancing, and accounting for medication adherence in clinical trials. Clin Pharmacol Ther 2014:95:617-26.

13 Anderson J, Peña AS, Sullivan T, et al. Does metformin improve vascular health in children with type 1 diabetes? Protocol for a one year, double blind, randomised, placebo controlled trial. BMC Pediatr 2013;13:108.

14 Anderson JJA, Couper JJ, Giles LC, et al. Effect of metformin on vascular function in children with type 1 diabetes: a 12-month randomized controlled trial. J Clin Endocrinol Metab 2017:102:4448-56.

15 Prevention CfDCa. Centres for Disease Control and Prevention Standardized Reference Charts. $2000 \mathrm{http}: / /$ wwwn.cdc.gov/epiinfo/.

16 Nichols GA, Rosales AG, Kimes TM, et al. Impact on glycated haemoglobin of a biological response-based measure of medication adherence. Diabetes Obes Metab 2015;17:843-8.

17 Raebel MA, Schmittdiel J, Karter AJ, et al. Standardizing terminology and definitions of medication adherence and persistence in research employing electronic databases. Med Care 2013;51(8 Suppl 3):S11-21.

18 Australia C. Australian Bureau of Statistics. Census of Population and Housing: Socio-Economic Indexes for Areas (SEIFA), cat. no. 2033.0.55.001 Australia: Australian Bureau of Statistics, 2011.

19 South Australian Department for Education. https://www.decd.sa.gov.au/teaching/ south-australian-state-schools-term-dates (Accessed 18 Dec 2018).

20 South Australia Public Holidays. http://www.publicholidaysa.com.au/ (Accessed 18 Dec 2017).

21 Ball R, Southern KW, McCormack P, et al. Adherence to nebulised therapies in adolescents with cystic fibrosis is best on week-days during school term-time. J Cyst Fibros 2013;12:440-4.

22 Ho PM, Rumsfeld JS, Masoudi FA, et al. Effect of medication nonadherence on hospitalization and mortality among patients with diabetes mellitus. Arch Intern Med 2006; 166:1836-41

23 Hood KK, Peterson CM, Rohan JM, et al. Association between adherence and glycemic control in pediatric type 1 diabetes: a meta-analysis. Pediatrics 2009;124:e1171-9.

24 King PS, Berg CA, Butner J, et al. Longitudinal trajectories of metabolic control across adolescence: associations with parental involvement, adolescents' psychosocial maturity, and health care utilization. J Adolesc Health 2012:50:491-6.

25 Helgeson VS, Siminerio L, Escobar O, et al. Predictors of metabolic control among adolescents with diabetes: a 4-year longitudinal study. J Pediatr Psychol 2009:34:254-70.

26 Marcovecchio ML, Chiesa ST, Bond S, et al. ACE Inhibitors and Statins in Adolescents with Type 1 Diabetes. N Engl J Med 2017;377:1733-45.

27 Nieuwlaat R, Wilczynski N, Navarro T, et al. Interventions for enhancing medication adherence. Cochrane Database Syst Rev 2014;11:Cd000011. 